\title{
Impact of prognostic nutritional index on long-term outcomes in patients with breast cancer
}

\author{
Tomomi Mohri', Yasuhiko Mohri ${ }^{2 *}$, Tsunehiko Shigemori $^{1}$, Kenji Takeuchi $^{1}$, Yoshiyuki Itoh $^{1}$ and Toshio Kato ${ }^{1}$
}

\begin{abstract}
Background: Prognostic nutritional index has been shown to be a prognostic marker for various solid tumors. However, few studies have investigated the impact of the prognostic nutritional index on survival of patients with breast cancer. The aim of this study was to investigate the impact of the prognostic nutritional index on the long-term outcomes in patients with breast cancer.

Methods: This study reviewed the medical records of 212 patients with breast cancer who underwent mastectomy. The prognostic nutritional index was calculated as $10 \times$ serum albumin $(\mathrm{g} / \mathrm{dl})+0.005 \times$ total lymphocyte count $\left(\right.$ per $\left.\mathrm{mm}^{3}\right)$. Receiver operating characteristic curve analysis was performed to determine the cutoff value of the prognostic nutritional index. The survival curves were calculated by the Kaplan-Meier method. Differences between the curves were analyzed by the log-rank test. Multivariate Cox proportional hazard model was used to evaluate the prognostic significance of prognostic nutritional index in patients with breast cancer.

Results: The mean prognostic nutritional index just before the operation was 51.9, and the median follow-up after surgery was 47.7 months. The optimal cutoff value of the prognostic nutritional index for predicting the overall survival was 52.8 from the receiver operating characteristic curve analysis. The 5 -year overall survival rate was 98 . $3 \%$ in the prognostic nutritional index $>52.8$ and $92.0 \%$ in the prognostic nutritional index $<52.8(P=0.013)$. In the multivariate analysis, a low prognostic nutritional index was an independent predictor for poor overall survival (HR, 5.88; $95 \% \mathrm{Cl}, 1.13-108.01 ; P=0.033)$.
\end{abstract}

Conclusions: The prognostic nutritional index is a simple and useful marker for predicting the long-term outcomes of breast cancer patients, independent of the tumor stage.

Keywords: Breast cancer, Prognostic nutritional index, Survival

\section{Background}

Breast cancer is the most frequently diagnosed malignancy and is the leading cause of cancer death among women [1]. The prognosis of breast cancer is influenced by wellrecognized host- and tumor-related factors, including patient age, histological type and grade, tumor size, lymph node status, estrogen receptor (ER) and progesterone receptor (PR) status, and human epidermal growth factor receptor 2 (HER2) status [2]. Despite recent improvements in early detection, progress in surgical techniques,

\footnotetext{
*Correspondence: ya-mohri@clin.medic.mie-u.ac.jp

${ }^{2}$ Department of Gastrointestinal and Pediatric Surgery, Mie University

Graduate School of Medicine, 2-174, Edobashi, Tsu city, Mie 514-0875, Japan

Full list of author information is available at the end of the article
}

chemotherapy, and endocrine therapy, some patients with breast cancer develop recurrence, even after curative resection. Therefore, accurate prediction of prognosis is needed to improve patient survival and to provide important information to the patients.

Serum albumin is one of the most commonly used markers for assessing nutritional status. Albumin is produced by the liver and is the major protein in blood, acting as a key antioxidant, detoxifier, and transporter of important nutrients. In advanced cancer patients, the levels of serum albumin fall sharply because malnutrition and systematic inflammatory response to tumors both suppress albumin synthesis [3]. The prevalence of malnutrition among breast cancer patients reported by 
two studies was $20.5 \%$ [4] and $18.3 \%$ [5], respectively. A Korean study also showed that $>51 \%$ of female breast cancer patients had moderate to high risk of malnutrition [6]. Malnutrition can cause many clinical consequences, including decreased quality of life, reduced treatment response, and increased treatmentrelated toxicity. The prognostic nutritional index (PNI), which is based on serum albumin concentration and total peripheral lymphocyte count, was originally proposed to assess the perioperative immunological status and surgical risk in patients undergoing gastrointestinal surgery [7]. Recently, the PNI has been shown to be a prognostic marker for various solid tumors [8-10]. However, few studies have investigated the impact of the PNI on survival of patients with breast cancer. Therefore, we retrospectively investigated the correlation between the PNI and clinicopathological factors and the impact of the PNI on survival in breast cancer patients.

\section{Methods \\ Patients}

A total of 219 patients with histologically confirmed breast cancer underwent surgery between January 2006 and October 2015 at the Department of Surgery, Toyama Hospital, Japan. We excluded seven patients with distant metastasis. Therefore, 212 patients were analyzed in this study. The median age was 66 (range 27-96) years. Most patients received adjuvant chemotherapy and/or endocrine therapy according to the clinical practice guidelines for breast cancer from the Japanese Breast Cancer Society [11], if necessary.

\section{Data collection}

Clinicopathological characteristics were obtained retrospectively from the medical records and evaluated as prognostic factors; these included patient age, tumor size, lymph node metastasis, pathological stage, hormonal receptor status (ER and PR), and HER2 status. The stage of breast cancer was classified according to the 7th edition of American Joint Committee on Cancer TNM Classification System [12].

We also collected data from blood tests just before the operation, including the level of serum albumin, total peripheral lymphocyte count, and carcinoembryonic antigen (CEA) and carbohydrate antigen (CA) 15-3 levels. PNI was calculated using the following formula: $10 \times$ serum albumin value $(\mathrm{g} / \mathrm{dl})+0.005 \times$ total lymphocyte count in the peripheral blood (per $\mathrm{mm}^{3}$ ).

\section{Statistical analysis}

The categorical variables were presented as numbers and percentages, and the groups were compared using the $\chi^{2}$ test. Continuous variables with normal distribution were expressed as the mean and standard deviation (SD) and were compared using the Mann-Whitney $U$ test or Kruskal-Wallis test.

At the time of the final follow-up (February 2016), the median follow-up was 47.7 months. Overall survival (OS) was defined as the time from the operation until death. Disease-free survival (DFS) was defined as the time from the operation to disease recurrence. In DFS analysis, the patients who died of any other cause rather than breast cancer were excluded. The survival curves were calculated by the Kaplan-Meier method. Differences between the curves were analyzed by the log-rank test. To evaluate the sensitivity and specificity for OS and DFS, the receiver operating characteristic (ROC) curve was calculated, and the Youden index was estimated to determine the optimal cutoff value for the PNI. Univariate associations with long-term survival were determined using Kaplan-Meier analysis and the logrank test. Multivariable analyses were performed by Cox proportional hazards regression analysis, incorporating

Table 1 Relationship between clinicopathological factors and PNI

\begin{tabular}{|c|c|c|c|c|}
\hline Variables & & $n(\%)$ & $\mathrm{PNI}$, mean $\pm \mathrm{SD}$ & $P$ value \\
\hline \multirow[t]{2}{*}{ Age (year) } & $\leqq 65$ & $102(48)$ & $53.1 \pm 4.1$ & $<0.001$ \\
\hline & $>65$ & $110(52)$ & $50.8 \pm 5.2$ & \\
\hline \multirow[t]{3}{*}{ Histology } & Ductal & $191(90)$ & $51.9 \pm 4.7$ & 0.291 \\
\hline & Lobular & $9(4)$ & $49.2 \pm 5.0$ & \\
\hline & Special & $12(6)$ & $53.4 \pm 6.2$ & \\
\hline \multirow[t]{3}{*}{ Tumor size $(\mathrm{cm})$} & $\leqq 2$ & $145(68)$ & $52.3 \pm 4.8$ & 0.166 \\
\hline & $>2, \leqq 5$ & $61(29)$ & $50.9 \pm 5.0$ & \\
\hline & $>5$ & $6(3)$ & $51.7 \pm 4.0$ & \\
\hline \multirow[t]{3}{*}{ Nodal metastasis } & 0 & $168(79)$ & $52.0 \pm 5.0$ & 0.240 \\
\hline & $1-3$ & $28(13)$ & $52.0 \pm 4.4$ & \\
\hline & $>3$ & $16(8)$ & $50.1 \pm 3.7$ & \\
\hline \multirow[t]{3}{*}{ Stage } & $0 / 1$ & $135(64)$ & $52.3 \pm 4.8$ & 0.124 \\
\hline & $\|$ & $58(27)$ & $51.5 \pm 4.9$ & \\
\hline & III & $19(9)$ & $50.5 \pm 4.5$ & \\
\hline \multirow{2}{*}{$\begin{array}{l}\text { Estrogen receptor } \\
\text { status }\end{array}$} & Positive & $168(79)$ & $51.8 \pm 5.0$ & 0.682 \\
\hline & Negative & $44(21)$ & $52.3 \pm 4.4$ & \\
\hline \multirow{2}{*}{$\begin{array}{l}\text { Progesterone receptor } \\
\text { status }\end{array}$} & Positive & $145(68)$ & $51.8 \pm 5.0$ & 0.879 \\
\hline & Negative & $67(32)$ & $52.1 \pm 4.6$ & \\
\hline \multirow[t]{2}{*}{ HER2 status } & Positive & $39(18)$ & $51.0 \pm 4.8$ & 0.152 \\
\hline & Negative & $173(82)$ & $52.1 \pm 4.8$ & \\
\hline \multirow[t]{2}{*}{ CEA (ng/ml) } & $<5$ & $164(77)$ & $51.9 \pm 4.6$ & 0.953 \\
\hline & $\geq 5$ & $48(23)$ & $51.9 \pm 5.5$ & \\
\hline \multirow[t]{2}{*}{ CA 15-3 (U/ml) } & $<23$ & $199(94)$ & $52.0 \pm 4.8$ & 0.327 \\
\hline & $\geq 23$ & $13(6)$ & $50.4 \pm 5.1$ & \\
\hline
\end{tabular}

$P N I$ prognostic nutritional index, $E R$ estrogen receptor, $P R$ progesterone receptor, HER-2 human epidermal growth factor receptor 2 
all variables with $P<0.10$ on univariate analysis. Statistical significance was defined as $P<0.05$. These analyses were conducted using JMP version 11.0.0 (SAS Institute, Cary, NC, USA).

\section{Results}

\section{PNI and clinicopathological characteristics of patients}

The mean PNI just before the operation was 51.9 (SD 4.9). Most of the 212 patients had ductal carcinoma, 67 (32\%) had a tumor $>2 \mathrm{~cm}$, and $44(21 \%)$ had axillary lymph node metastasis. The mean PNI in patients aged $>65$ years was significantly lower than that in patients aged $\leqq 65$ years $(P<0.001$; Table 1$)$. The PNI value gradually decreased with the advancing disease stage, but significant difference was not observed $(P=0.124$; Table 1$)$.

\section{ROC analysis}

Using OS as an endpoint, the area under the ROC curve for the PNI was 0.676. When the PNI was 52.8, the Youden index was maximal, with a sensitivity of $91.7 \%$ and specificity of $41.5 \%$. Therefore, the cutoff value of the PNI was set at 52.8. Then, 85 patients (40.0 \%) with a PNI $>52.8$ and 127 patients $(60.0 \%)$ with a PNI $\leq 52.8$ were classified into PNI-high and PNIlow groups, respectively. During the studied period, four patients died of any other cause rather than breast cancer. Those patients were excluded from DFS analysis. Using DFS as an endpoint, the area under the ROC curve for the PNI was 0.657. The cutoff value of the PNI on DFS was set at 52.4. There were 90 patients with a PNI $>52.4$ (a PNI-high group) and 118 patients with a PNI $\leq 52.4$ (a PNI-low group).

\section{OS and DFS}

The 5-year OS rate was $98.3 \%$ in the PNI-high group and $92.0 \%$ in the PNI-low group $(P=0.019$; Fig. 1a). One patient $(1.2 \%)$ died in the PNI-high group, and seven $(5.5 \%)$ died in the PNI-low group. The cause of death in the PNI-low group was tumor relapse in four patients, other cancer in two patients, and a cause other than cancer in one patient. One patient in PNI-high group died of a cause other than cancer. The 5-year DFS rate was $97.1 \%$ in the PNI-high group and $92.0 \%$ in the PNI-low group $(P=0.035$; Fig. $1 b)$.

\section{Predictive factors for OS}

In the univariate analysis, tumor size, lymph node metastasis, and PNI were significantly associated with OS (Table 2). The multivariate analysis demonstrated that PNI and lymph node metastasis were independent prognostic factors for the OS (Table 3).

\section{Discussion}

Our present study demonstrated that the PNI can predict the long-term outcomes of breast cancer patients, independent of the conventional TNM classification. The OS and DFS rates of the PNI-low group were significantly lower than those of the PNI-high group. The multivariate analysis performed in the present study demonstrated that the PNI was an independent predictor for the OS.

There are several known predictors of breast cancer prognosis, such as tumor size, histological type, lymph node involvement, hormonal receptor status, and HER2 status. However, it is of interest that there are other host-related factors, such as the PNI. Previous studies have reported an impact of the PNI on the long-term outcomes in several solid tumors, and various cutoff values for the PNI were used in those studies [8-10]. The cutoff value was usually set at 45 , because PNI $<45$ is defined as moderate to severe malnutrition. However, the optimal cutoff value of the PNI to predict the longterm outcomes remains unclear. In the present study, we performed a ROC curve analysis, and the optimal cutoff value for the PNI was determined to be 52.8 . When the PNI was 52.8, the sensitivity and specificity
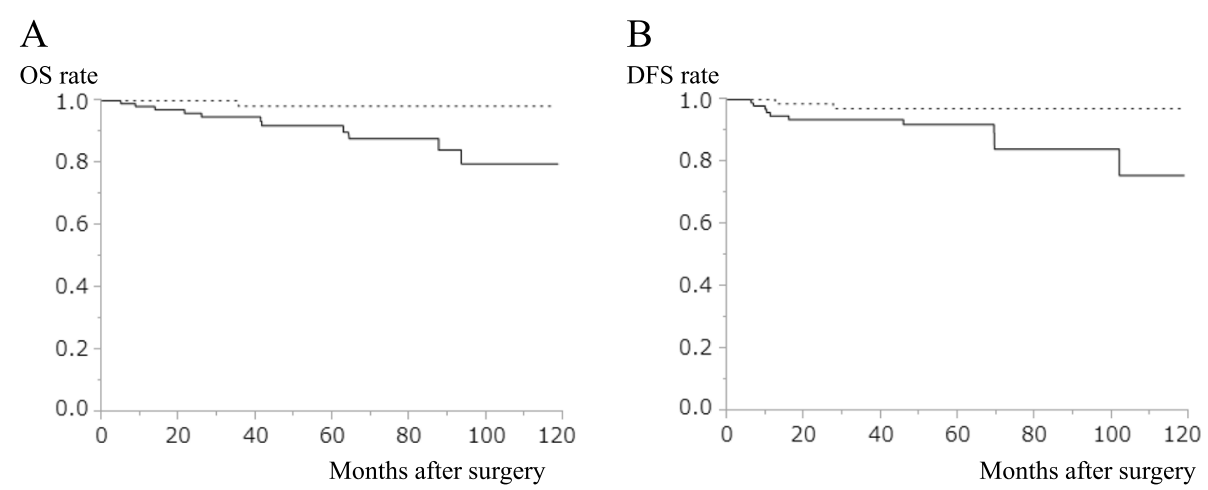

Fig. 1 Kaplan-Meier estimates of OS and DFS according to the PNI. a OS rate of the PNI-low group was significantly lower than that of the PNIhigh group $(P=0.019)$. b DFS rate of the PNI-low group was significantly lower than that of the PNI-high group $(P=0.035)$. Solid line indicates the $\mathrm{PNI}-$ low group, and dotted line indicates the PNI-high group 
Table 2 Univariate analysis of prognostic factors for OS

\begin{tabular}{llll}
\hline Variables & & 5 -year survival (\%) & $P$ value \\
\hline Age (year) & $\leqq 65$ & 100 & $<0.001$ \\
& $>65$ & 88.4 & \\
Histological type & Ductal & 94.8 & 0.174 \\
& Lobular/ & 93.8 & \\
& Special & & \\
Tumor size (cm) & $\leq 2$ & 95.9 & 0.005 \\
& $>2$ & 91.7 & \\
Lymph node metastasis & Negative & 95.7 & 0.001 \\
& Positive & 90.4 & \\
Estrogen receptor status & Negative & 94.3 & 0.827 \\
& Positive & 94.8 & \\
Progesterone receptor status & Negative & 92.5 & 0.619 \\
& Positive & 95.9 & \\
HER2 status & Negative & 95.3 & 0.967 \\
& Positive & 90.8 & \\
CEA (ng/ml) & $<5$ & 93.7 & 0.152 \\
& $\geq 5$ & 97.7 & \\
CA15-3 (U/ml) & $\geq 23$ & 94.9 & 0.101 \\
PNI & $\geq 23$ & 88.9 & 0.019 \\
& $>52.8$ & 98.3 & \\
& $\leq 52.8$ & 92.0 &
\end{tabular}

OS overall survival, ER estrogen receptor, $P R$ progesterone receptor, HER-2 human epidermal growth factor receptor 2, CEA carcinoembryonic antigen, $C A$ 15-3 carbohydrate antigen 15-3, PNI prognostic nutritional index

for the 5-year OS were 91.7 and $41.5 \%$, respectively. Few studies have determined the prognostic impact of the PNI in breast cancer patients. Only one study has shown that the preoperative PNI can be used as a simple and useful marker for predicting the long-term outcomes of triple-negative breast cancer [13]. The present study showed that a low preoperative PNI was associated with a higher risk of postoperative recurrence of breast cancer independent of hormonal receptor status and TNM stage.

The association between decreased PNI and poor survival of breast cancer is probably complex and largely unclear; however, possible explanations do exist. PNI is derived from the absolute albumin and absolute lymphocyte counts and is a routinely available laboratory test. One potential mechanism underlying the prognostic impact of PNI is that low PNI reflects hypoalbuminemia. Serum albumin has been used to assess disease severity, progression, and prognosis. Another factor is that lymphocytes play an important role in the host

Table 3 Cox proportional multivariate hazard models for OS

\begin{tabular}{llclc}
\hline Variables & & Hazard ratio & $95 \% \mathrm{Cl}$ & $P$ value \\
\hline Age & $>65$ & 11.9 & $2.23-220.81$ & 0.002 \\
Tumor size & $>2 \mathrm{~cm}$ & 1.94 & $0.45-8.91$ & 0.377 \\
Lymph node metastasis & Positive & 4.06 & $1.05-17.86$ & 0.042 \\
PNI & $\leqq 52.8$ & 5.88 & $1.13-108.01$ & 0.033 \\
\hline
\end{tabular}

$\mathrm{Cl}$ confidence interval, $\mathrm{PNI}$ prognostic nutritional index immune response to eradicate the formation and progression of tumors [14]. High lymphocytic infiltration is associated with improved survival, independent of clinicopathological characteristics, in primary operable ductal invasive breast cancer [15]. The importance of lymphocytes has been highlighted in several studies in which increasing infiltration of tumors with lymphocytes was associated with better response to cytotoxic treatment and prognosis in breast cancer patients [16-18]. Thus, low PNI may confer a survival advantage by tumor cells and lead to poorer outcome and increased recurrence.

The major limitations of the present study were the retrospective nature of the study and the single-center design. We were unable to exclude the possibility that unequal distribution of unidentified clinicopathological parameters in our patient cohort may have biased the results. Therefore, a large, prospective study should therefore be performed to confirm our findings.

\section{Conclusions}

Our clinical observation shows that the PNI is associated with OS and DFS in patients with breast cancer. The PNI is an easy-to-determine, reproducible, and inexpensive test. It can be easily incorporated into routine use as a prognostic factor. Despite all these results, prospective studies evaluating PNI in a large series are required in this field.

\section{Additional file}

Additional file 1: The raw data of this study.

\section{Abbreviations}

CA, carbohydrate antigen; CEA, carcinoembryonic antigen; DFS, disease-free survival; ER, estrogen receptor; HER2, human epidermal growth factor receptor 2; OS, overall survival; PNI, prognostic nutritional index; PR, progesterone receptor; $R O C$, receiver operating characteristic; SD, standard deviation.

\section{Acknowledgements}

Not applicable.

Funding

The authors declare that they had no funding source for this study.

\section{Availability of data and materials}

The dataset supporting the conclusions of this article is included within the article (Additional file 1).

\section{Authors' contributions}

TM designed the research and wrote the manuscript. TS, KT, and YI collected the data. TM, YM, and TK analyzed the data and performed the statistical analysis. YM reviewed and edited the manuscript. All authors read and approved the final manuscript.

\section{Competing interests}

The authors declare that they have no competing interests.

Consent for publication

Not applicable. 


\section{Ethics approval and consent to participate}

This study was conducted in accordance with the guidelines of the 1975 Declaration of Helsinki. The need for informed patient consent was waived because of the retrospective nature of the study. The study protocol has been approved by the ethics committee of Toyama Hospital (no. 271026).

\section{Author details}

'Department of Surgery, Toyama Hospital, Tsu city, Mie 514-0043, Japan.

${ }^{2}$ Department of Gastrointestinal and Pediatric Surgery, Mie University

Graduate School of Medicine, 2-174, Edobashi, Tsu city, Mie 514-0875, Japan.

Received: 21 December 2015 Accepted: 15 June 2016

Published online: 27 June 2016

\section{References}

1. Siegel R, Ma J, Zou Z, Jemal A. Cancer statistics, 2014. CA Cancer J Clin. 2014;64:9-29.

2. Lal P, Tan LK, Chen B. Correlation of HER-2 status with estrogen and progesterone receptors and histologic features in 3,655 invasive breast carcinomas. Am J Clin Pathol. 2005;123:541-6.

3. Yeun JY, Kaysen GA. Factors influencing serum albumin in dialysis patients. Am J Kidney Dis. 1998;32(Supplement 4):S118-25.

4. Hébuterne X, Lemarié $E$, Michallet $M$, de Montreuil CB, Schneider SM, Goldwasser F. Prevalence of malnutrition and current use of nutrition support in patients with cancer. J Parenter Enteral Nutr. 2014;38:196-204.

5. Pressoir M, Desné S, Berchery D, Rossignol G, Poiree B, Meslier M, Traversier S, Vittot M, Simon M, Gekiere JP, Meuric J, Serot F, Falewee MN, Rodrigues I, Senesse P, Vasson MP, Chelle F, Maget B, Antoun S, Bachmann P.

Prevalence, risk factors and clinical implications of malnutrition in French Comprehensive Cancer Centres. Br J Cancer. 2010;102:966-71.

6. Wie GA, Cho YA, Kim SY, Kim SM, Bae JM, Joung H. Prevalence and risk factors of malnutrition among cancer patients according to tumor location and stage in the National Cancer Center in Korea. Nutrition. 2010;26:263-8.

7. Onodera T, Goseki N, Kosaki G. Prognostic nutritional index in gastrointestinal surgery of malnourished cancer patients. Nihon Geka Gakkai Zasshi. 1984;85:1001-5 (Japanese)

8. Pinato DJ, North BV, Sharma R. A novel, externally validated inflammationbased prognostic algorithm in hepatocellular carcinoma: the prognostic nutritional index (PNI). Br J Cancer. 2012;106:1439-45.

9. Mohri Y, Inoue Y, Tanaka K, Hiro J, Uchida K, Kusunoki M. Prognostic nutritional index predicts postoperative outcome in colorectal cancer. World J Surg. 2013;37:2688-92.

10. Sun $\mathrm{K}$, Chen $\mathrm{S}, \mathrm{Xu}$ J, Li G, He Y. The prognostic significance of the prognostic nutritional index in cancer: a systematic review and metaanalysis. J Cancer Res Clin Oncol. 2014;140:1537-49.

11. Mukai H, Aihara T, Yamamoto Y, Takahashi M, Toyama T, Sagara Y, Yamaguchi H, Akabane H, Tsurutani J, Hara F, Fujisawa T, Yamamoto N, Ohsumi S. The Japanese Breast Cancer Society Clinical Practice Guideline for systemic treatment of breast cancer. Breast Cancer. 2015;22:5-15.

12. Greene FL. Breast tumours. In: Sobin LH, Gospodarowicz MK, Wittekind C, editors. TNM classification of malignant tumours. 7th ed. Oxford: WileyBlackwell; 2009. p. 181-93.

13. Yang Z, Zhang B, Hou L, Xie $Y$, Cao X. Pre-operative prognostic nutritional index predicts the outcomes for triple-negative breast cancer. Tumour Biol. 2014;35:12165-71.

14. Hanahan D, Weinberg RA. Hallmarks of cancer: the next generation. Cell. 2011;144:646-74

15. Murri AM, Hilmy M, Bell J, Wilson C, McNicol AM, Lannigan A, Doughty JC, McMillan DC. The relationship between the systemic inflammatory response, tumour proliferative activity, T-lymphocytic and macrophage infiltration, microvessel density and survival in patients with primary operable breast cancer. Br J Cancer. 2008:99:1013-9.

16. Loi S, Sirtaine N, Piette F, Salgado R, Viale G, Van Eenoo F, et al. Prognostic and predictive value of tumor-infiltrating lymphocytes in a phase III randomized adjuvant breast cancer trial in node-positive breast cancer comparing the addition of docetaxel to doxorubicin with doxorubicinbased chemotherapy: BIG 02-98. J Clin Oncol. 2013;31:860-7.
17. Gooden MJ, de Bock GH, Leffers N, Daemen T, Nijman HW. The prognostic influence of tumour-infiltrating lymphocytes in cancer: a systematic review with meta-analysis. Br J Cancer. 2011;105:93-103.

18. Denkert C, Loibl S, Noske A, Roller M, Müller BM, Komor M, Budczies J, DarbEsfahani S, Kronenwett R, Hanusch C, von Törne C, Weichert W, Engels K, Solbach C, Schrader I, Dietel M, von Minckwitz G. Tumor-associated lymphocytes as an independent predictor of response to neoadjuvant chemotherapy in breast cancer. J Clin Oncol. 2010;28:105-13.

\section{Submit your next manuscript to BioMed Central and we will help you at every step:}

- We accept pre-submission inquiries

- Our selector tool helps you to find the most relevant journal

- We provide round the clock customer support

- Convenient online submission

- Thorough peer review

- Inclusion in PubMed and all major indexing services

- Maximum visibility for your research

Submit your manuscript at www.biomedcentral.com/submit
) Biomed Central 